

\title{
Exploring AGN Activity over Cosmic Time with the SKA
}

\author{
Vernesa Smolčić ${ }^{* 1}$, Paolo Padovani ${ }^{2}$, Jacinta Delhaize ${ }^{1}$, Isabella Prandoni ${ }^{3}$, \\ Nicholas Seymour $^{4}$, Matt Jarvis ${ }^{5,6}$, Jose Afonso ${ }^{7,8}$, Manuela Magliocchetti ${ }^{9}$ \\ Minh Huynh $^{10}$, Mattia Vaccari ${ }^{6}$, Alexander Karim ${ }^{11}$ \\ ${ }^{1}$ University of Zagreb, Physics Department, Bijenička cesta 32, 10002 Zagreb, Croatia; \\ ${ }^{2}$ European Southern Observatory, Karl-Schwarzschild-Strasse 2, 85748 Garching b. München, \\ Germany; ${ }^{3}$ INAF - Istituto di Radioastronomia, Bologna, Italy; ${ }^{4}$ International Centre for Radio \\ Astronomy Research, Curtin University, Perth, Australia; ${ }^{5}$ Astrophysics, University of Oxford, \\ Keble Road, Oxford, OX1 3RH, UK, ${ }^{6}$ Physics Department, University of the Western Cape, \\ Bellville 7535, South Africa; ${ }^{7}$ Instituto de Astrofísica e Ciências do Espaço, Universidade de \\ Lisboa, OAL, Tapada da Ajuda, PT1349-018 Lisboa, Portugal; ${ }^{8}$ Departamento de Física, \\ Faculdade de Ciências, Universidade de Lisboa, Edifício C8, Campo Grande, PT1749-016 \\ Lisbon, Portugal; ${ }^{9}$ INAF-IAPS, Via Fosso del Cavaliere 100, I-00133 Roma, Italy; \\ ${ }^{10}$ International Center for Radio Astronomy Research, M468, University of Western Australia, \\ Crawley, WA 6009, Australia; ${ }^{11}$ Argelander Institut for Astronomy, Auf dem Hügel 71, Bonn \\ D-53121, Germany \\ E-mail: vs@phy.hr
}

In this Chapter we present the motivation for undertaking both a wide and deep survey with the SKA in the context of studying AGN activity across cosmic time. With an rms down to $1 \mu \mathrm{Jy} /$ beam at $1 \mathrm{GHz}$ over $1,000-5,000 \mathrm{deg}^{2}$ in 1 year (wide tier band 1/2) and an rms down to $200 \mathrm{nJy} /$ beam over $10-30 \mathrm{deg}^{2}$ in 2000 hours (deep tier band 1/2), these surveys will directly detect faint radio-loud and radio-quiet AGN (down to a $1 \mathrm{GHz}$ radio luminosity of about $2 \times$ $10^{23} \mathrm{~W} / \mathrm{Hz}$ at $z=6$ ). For the first time, this will enable us to conduct detailed studies of the cosmic evolution of radio AGN activity to the cosmic dawn $(z \gtrsim 6)$, covering all environmental densities.

Advancing Astrophysics with the Square Kilometre Array

June 8-13, 2014

Giardini Naxos, Italy

\footnotetext{
* Speaker.
} 


\section{Introduction}

Galaxies are thought to evolve over time from an initial stage of blue star forming galaxies with spiral morphology towards quiescent red galaxies with spheroidal morphologies and the highest stellar masses (e.g., Faber et al. 2007). A galaxy evolves through interspersed episodes of intensive mass accretion onto the stellar body, as well as the central super-massive black hole (SMBH), creating a powerful active galactic nucleus (AGN; Sanders \& Mirabel 1996). This is consistent with the $\Lambda \mathrm{CDM}$ paradigm, in which structure in the Universe grows hierarchically in such a way that small structures evolve into larger ones. In this context, faint $\left(\mathrm{L}_{1.4 \mathrm{GHz}}<10^{25} \mathrm{~W} / \mathrm{Hz}\right)$ but still radio-loud AGN remain puzzling. ${ }^{1}$ These faint radio loud AGN are found in red, quiescent galaxies that would not be identified as AGN at any other wavelength (e.g., Hickox et al. 2009) and they do not seem to fit into the Unified Model for AGN (e.g., Hardcastle et al. 2007). They often reside at the bottom of the galaxy cluster/group potential wells and their radio-bright outflows heat the intra-cluster/group gas and the hot gas halo of the host galaxy (e.g. Fabian 2012; Best et al. 2006). This heating, deemed crucial in cosmological models of galaxy formation, has been termed 'radiomode' feedback (Granato et al. 2004; Croton et al. 2006; Bower et al. 2006). However, both on group/cluster and galaxy scales, feedback is still poorly understood. ${ }^{2}$

In the context of the most powerful AGN, Type 1 (broad line) AGN (quasars hereafter), are a population that experiences the most intense SMBH growth. Quasar winds associated with this intense SMBH growth are thought to quench their galactic star formation by expelling a fraction of the interstellar gas (so called 'quasar mode AGN feedback'; e.g. Hopkins et al. 2006). The existence of two, physically distinct, radio-loud (RL hereafter) and radio-quiet (RQ hereafter) ${ }^{3}$ quasar populations is a long debated issue that has far-reaching implications for astrophysical models, including unified models for AGN and the evolution of star formation. Although the quasar radioloudness distribution has been carefully studied in many different quasar samples over the past few decades (e.g. Strittmatter et al. 1980; Ivezić et al. 2002; White et al. 2000, 2007; Cirasuolo et al. 2003; Baloković et al. 2012), there is still no definite understanding or consensus. The bimodality could imply two physically distinct types of quasars (pointing to e.g. different SMBH accretion/spin mechanisms or physically different sources of synchrotron emission) or be due to differing geometries (e.g. Fanidakis et al. 2011). Furthermore, recent studies suggest that, in general, radio emission in radio-quiet AGN may arise from star formation related processes (e.g. Kimball et al. 2011; Padovani et al. 2011; Condon et al. 2012; Bonzini et al. submitted). However,

\footnotetext{
${ }^{1}$ For Type 1 AGN we here define radio loudness $\left(R^{\prime}\right)$ following White et al. (2000) and Ivezić et al. (2002) as the logarithm of the ratio of radio-to-optical fluxes: $R^{\prime}=\log \frac{F_{\text {radio }}}{F_{\text {optical }}}=0.4(i-t)$ where $i$ is the optical $i$-band AB magnitude and $t=-2.5 \log \left(\frac{F_{1.4 \mathrm{GHz}}}{3631 \mathrm{Jy}}\right)$ is the $\mathrm{AB}$ magnitude at $1.4 \mathrm{GHz}$. The adopted radio-loud vs. radio-quiet threshold is $R^{\prime}=1$. For Type 2 AGN we define radio-loud sources as those with $q=\log \left[\left(F_{\mathrm{FIR}} / 3.75 \times 10^{12}\right) / F_{1.4 \mathrm{GHz}}\right]<1.7$, where $F_{\mathrm{FIR}}$ is the far-IR flux between $42.5 \mu \mathrm{m}$ and $122.5 \mu \mathrm{m}$ (e.g., Machalski \& Condon 1999; Padovani et al. 2011) .

${ }^{2}$ We note that powerful $\left(\mathrm{L}_{1.4 \mathrm{GHz}}>10^{25} \mathrm{~W} / \mathrm{Hz}\right)$, high-excitation AGN, that fit into the Unified model for AGN, also can exert feedback through their jets (e.g. Shabala et al. 2011; Zakamska \& Greene 2014). However, cosmological models assume that 'radio-mode' feedback is cosmologically important through feedback exerted by galaxies that have reached their 'quiescent' phase of black-hole accretion (e.g. Croton et al. 2006). This 'quiescent' galaxy phase is directly linked to low-excitation radio AGN (Smolčić 2009) predominantly found at low radio luminosities (e.g. Kauffmann et al. 2008; Best \& Heckman 2012).

${ }^{3}$ We here refer to radio luminous Type 1 , broad line AGN as quasars.
} 
this is contradicted by White et al. (2014), who find evidence for black-hole accretion still making a significant contribution to the total radio emission. We note that such studies are subject to biases in terms of the luminosity and redshift ranges studied. For example, the deep-field work of Bonzini et al. (submitted) is sensitive to fainter AGN which may be hosted in spiral galaxies which are likely to have ongoing star formation, whereas the wide-field work of Kimball et al. (2011) and Condon et al. (2012) detect the more luminous AGN, which are more likely to be hosted by massive ellipticals (e.g. Dunlop et al. 2003).

In extragalactic radio surveys, two dominant galaxy populations are observed. These are AGN and star forming galaxies. At the bright end ( $21 \mathrm{mJy}$ ), the $\mathrm{GHz}$ radio-bright sky consists mainly of "classical" radio AGN, i.e. radio quasars and radio galaxies. Their radio emission is generated from the gravitational energy associated with a SMBH and emitted through relativistic jets of particles as synchrotron radiation. Below $1 \mathrm{mJy}$ there is an increasing contribution to the radio source population from massive star formation. In this case, synchrotron emission is produced via relativistic plasma ejected from supernovae. However, such star forming galaxies (SFGs) appear not to be the only component of the faint radio sky, at least down to $\sim 50 \mu \mathrm{Jy}$ at a few $\mathrm{GHz}$ (e.g., Gruppioni et al. 2003; Jarvis \& Rawlings 2004; Simpson et al. 2006a; Smolčić et al. 2008; Mignano et al. 2008; Padovani et al. 2009). At the faint radio levels $(<1 \mathrm{mJy})$ the source counts are still well populated by both RQ and RL AGN.

Sensitive radio continuum surveys, as will be provided with the SKA1 wide and deep tier band 1/2 surveys, are of extreme relevance for a variety of reasons: (1) Only deep radio observations trace AGN hosted by otherwise quiescent galaxies, thought to be the main drivers of the radiomode feedback; (2) The least luminous RQ AGN reside typically in spiral galaxies, which are still forming stars, and therefore are likely to provide a vital contribution to our understanding of AGN - galaxy co-evolution; (3) Radio observations are unaffected by absorption and therefore sensitive to all types of AGN, independently of obscuration and their orientation (i.e., Type 1s and Type 2s); (4) Finally and most importantly, sensitive radio observations, that only the new-generation radio interferometers can provide, will start to detect the bulk ( $~ 90 \%)$ of the AGN population, currently missed by the majority of existing radio surveys (e.g. Ivezić et al. 2002; Baloković et al. 2012).

For the remainder of the Chapter we assume the following SKA1 deep and wide tier band $1 / 2$ survey characteristics. For the wide survey we assume an $\mathrm{rms}$ of $\sim 1 \mu \mathrm{Jy} / \mathrm{beam}$ over 1,000 $-5,000 \mathrm{deg}^{2}$ reached in 1 year of observations, and for the deep survey we assume an rms of $0.2 \mu \mathrm{Jy} /$ beam over $10-30 \mathrm{deg}^{2}$ for 2,000 hours of observations at an observing frequency of $1 \mathrm{GHz}$ (see Prandoni \& Seymour 2015, for more details on the surveys).

\section{AGN activity in the faint radio sky}

Radio source counts are the most straight-forward information drawn from a radio survey and are commonly used to predict source counts in future deeper surveys. They flatten below $1 \mathrm{mJy}$ and are generally expected to decrease again at fainter fluxes (e.g. Hopkins et al. 2000; Wilman et al. 2008; Condon et al. 2012). In Fig. 1 we show a compilation of various survey results and the simulated results for presently unreached levels, separated for RL and RQ AGN. Various methods, all relying on multi-wavelength data, have been employed to separate the radio faint population into star forming and AGN galaxies (e.g. Smolčić et al. 2008; Seymour et al. 2008; Simpson et al. 


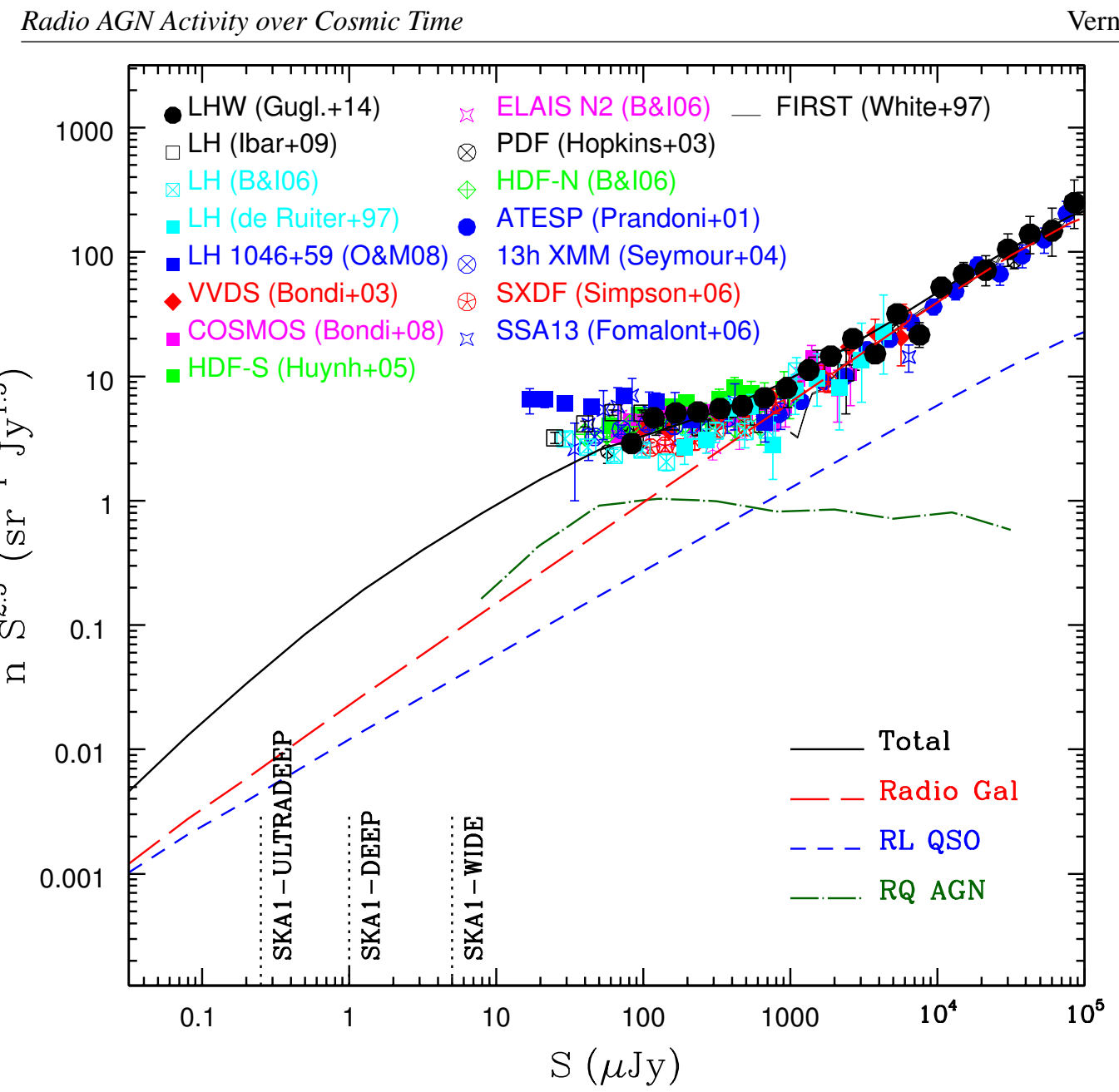

Figure 1: A compilation of observed radio source counts and simulated counts, divided into radio-loud quasars, radio-quiet AGN, and radio galaxies (as indicated in the legend, the data are taken from Guglielmino et al., in preparation; Ibar et al. 2009; Biggs \& Ivison 2006; de Ruiter et al. 1997; Owen \& Morrison 2008; Bondi et al. 2003, 2008; Huynh et al. 2005; Biggs \& Ivison 2006; Hopkins et al. 2003; Prandoni et al. 2001; Seymour et al. 2004; Simpson et al. 2006b; Fomalont et al. 2006; White et al. 1997). The simulated counts are taken from the SKADS model (Wilman et al. 2008). Also indicated are the $5 \sigma$ SKA1 wide, deep and Ultradeep limits.

2012; McAlpine et al. 2013; Bonzini et al. 2013). For example, using optical, IR, X-ray and radio data, Bonzini et al. (2013) disentangle the SFG, RQ, and RL AGN in the Extended Chandra deep Field South (ECDFS) survey ( $\sim 6 \mu \mathrm{Jy}$ rms noise in a $2.8 "$ x $1.6 "$ beam over $0.3 \mathrm{deg}^{2}$ containing 900 radio sources; see also Miller et al. 2008, 2013; Bonzini et al. 2012; Padovani et al. 2011).

Fig. 2, adapted from Bonzini et al. (2013), shows the relative fractions of the various radio source classes as a function of radio flux density. As expected, AGN dominate at large flux densities ( $\gtrsim 1 \mathrm{mJy}$ ). Above $\sim 0.1 \mathrm{mJy}$ RL AGN are the predominant type of AGN, however their fractional contribution steeply decreases towards lower flux densities. On the other hand, below $0.1 \mathrm{mJy}$ the radio sky is dominated by star-formation-related processes, rather than RL and/or RQ AGN. Thus, deep observations of large areas of the sky are needed to assemble statistically-sound samples of the faintest RQ and RL AGN and to study their physical properties and cosmic evolution. Such surveys will be provided by SKA1 and SKA2. At the above assumed depth and area of the wide (5000 deg ${ }^{2}$, 




Figure 2: Relative fraction of the various classes of E-CDFS sources as a function of radio flux density: SFG (green diamonds), all AGN (magenta triangles), radio-quiet AGN (blue circles) and radio-loud AGN (red squares). Adapted from Bonzini et al. (2013).

$\mathrm{rms} \sim 1 \mu \mathrm{Jy} /$ beam $)$ and deep (30 $\mathrm{deg}^{2}, \mathrm{rms} \sim 0.2 \mu \mathrm{Jy} /$ beam $)$ tier SKA1 surveys, about $3 \times 10^{7}$ and $4 \times 10^{5}$ AGN, respectively, are predicted using the SKADS (Wilman et al. 2008, 2010) simulations of the extragalactic radio sky based on models of the evolution of the radio luminosity function. This is orders of magnitude larger than the number of sources detected in the deepest radio surveys to-date, e.g. COSMOS (rms $\sim 10-15 \mu \mathrm{Jy} /$ beam, $2 \mathrm{deg}^{2}, \sim 2,500$ sources, Schinnerer et al. 2007, 2010) and ECDFS (rms $\sim 6 \mu \mathrm{Jy} / \mathrm{beam}, 0.3 \mathrm{deg}^{2}, \sim 900$ sources, Miller et al. 2008, 2013). For comparison, MeerKAT-MIGHTEE ( $\mathrm{rms} \sim 1 \mu \mathrm{Jy} / \mathrm{beam}, 35 \mathrm{deg}^{2}, 1.4 \mathrm{GHz}$ ) is expected to detect about 174,000 AGN, while ASKAP-EMU (rms $\sim 10 \mu \mathrm{Jy} / \mathrm{beam}, 30,000 \mathrm{deg}^{2}, 1.4 \mathrm{GHz}$ ) will detect $\sim 2.8 \times 10^{7} \mathrm{AGN}$.

\subsection{Radio emission in radio-quiet AGN}

RQ AGN are characterized by relatively low radio-to-optical flux density ratios and radio powers and, until recently, have been found predominantly in optically selected samples. We note that the distinction between RQ and RL AGN is not simply a matter of semantics - the two classes represent intrinsically different objects. RL AGN emit most of their energy over the entire electromagnetic spectrum non-thermally and in association with powerful relativistic jets. The multiwavelength emission of RQ AGN is dominated by thermal emission and is related to the accretion disk. The exact mechanism responsible for radio emission in RQ AGN has been a matter of debate for the past fifty years. Explanations have included, for example, a scaled down version of the RL AGN mechanism (e.g., Miller et al. 1993; Ulvestad et al. 2005), and star formation (Sopp \& Alexander 1991).

There is still no clear consensus on the existence of a bimodality in the radio-loudness distribution of quasars. A bimodality would imply two physically distinct types of quasars in the Universe and would point to, for instance, different SMBH accretion/spin mechanisms, geometries or physical properties (e.g. Fanidakis et al. 2011; Kimball et al. 2011; Condon et al. 2013). This issue is still open mainly due to the overwhelmingly high fraction of RQ quasars that regularly go undetected 
in radio surveys. As shown in Fig. 3 all current radio surveys (even the deepest ones) barely sample even the loudest end of the radio-quiet part of the distribution. Hence, as only $\sim 10 \%$ of optically selected quasars are RL (e.g. Ivezić et al. 2002), this means that a major fraction of quasars still remains undetected and unexplored at radio wavelengths (see also Baloković et al. 2012). Only observations of large sky areas to the depths reachable with the SKA1 deep and wide surveys will directly reveal the radio properties of roughly $90 \%$ of optically detected quasars.

Recent studies of radio-quiet AGN suggest different radio emitting mechanisms in RL and RQ AGN (e.g. Kimball et al. 2011; Padovani et al. 2011; Condon et al. 2013; Bonzini et al. 2013). For example, investigating the cosmic evolution of RQ AGN and SFGs, the results presented in Padovani et al. (2011) suggest very close ties between star formation and radio emission in RQ AGN at $z \sim 1.5-2$. They find that the evolution of RQ AGN is similar to that of SFG (see also Smolčić et al. 2009b, and references therein) and that their luminosity function appears to be an extension of the SFG LF (see also Kimball et al. 2011; Condon et al. 2013). If RQ AGN were simply scaled-down versions of RL AGN, it could be expected that they share the evolutionary properties of the latter and their luminosity function should also be an extrapolation of the RL luminosity function at low powers, however this does not appear to be the case (e.g. Fernandes et al. 2011). This has prompted studies of the emission mechanism of synchrotron radiation from RQ AGN. For example, Kimball et al. (2011); Padovani et al. (2011); Condon et al. (2013); Bonzini et al. (2013) suggest that star formation in the host galaxy of RQ AGN may be the dominant contributor to the radio continuum emission, whereas White et al. (2014) compare the radio emission from a sample of faint radio-quiet quasars with the massive galaxy population, and suggest that the radio emission in optically-selected radio-quiet quasars is consistent with being due to the AGN. These studies envelope different luminosity (both optical and radio) and redshift ranges, and some of the differences may be attributable to to the diversity of the samples. Furthermore, a close link between star formation and radio emission in RQ AGN is further affirmed by the comparison of the star formation rates (SFRs) derived from the far-IR luminosities and the radio luminosities, assuming that all the radio emission is due to star formation (Bonzini et al. submitted). For RQ AGN and SFGs, the two SFR estimates are consistent. For RL AGN, the agreement is poor due to the large contribution of the relativistic jet to their radio luminosity (Morić et al. 2010; Bonzini et al. submitted). Another intriguing possibility is that both AGN and SF processes contribute to the total radio (and IR) emission, in some relative proportion (e.g. Morić et al. 2010). Seyfert 2 galaxies are a well-established example in the local Universe (e.g. Roy et al. 1998) and recent studies indicate that composite AGN/SF systems may constitute a significant fraction of the galaxy population at high redshifts (e.g. Daddi et al. 2007; Gruppioni et al. 2011; Del Moro et al. 2013).

The SKA1 wide and deep surveys, in conjunction with multi-wavelength data will provide the basis to resolve the long-standing quasar radio-loudness dichotomy, and the possible interplay between coexisting AGN and star formation phenomena. However, only once star-formation and AGN activity are reliably separated, and/or their fractional contribution to individual sources is determined, it is possible to derive unbiased radio luminosity functions and disentangle the contributions of each type of activity over cosmic time via direct radio detections and stacking in the radio map (e.g. Smolčić et al. 2008; Karim et al. 2011; Zwart et al. 2014). 

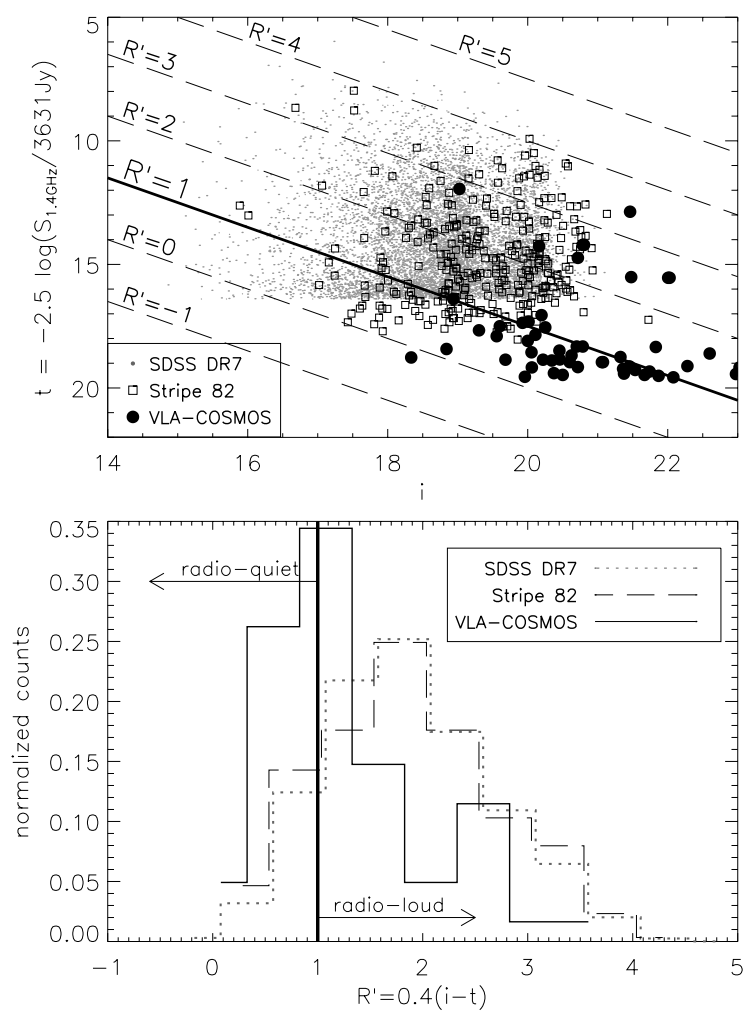

Figure 3: Top: $i$-band vs. $1.4 \mathrm{GHz}$ radio magnitude $(t)$ distribution for optically selected quasars (i.e. broad line AGN) drawn from current state-of-the-art surveys: SDSS DR7 - FIRST $\left(\sim 9,000 \mathrm{deg}^{2}, \mathrm{~S}_{1.4 \mathrm{GHz}} \gtrsim 1 \mathrm{mJy}\right.$; Schneider et al. 2010), Stripe $82\left(92 \mathrm{deg}^{2}, \mathrm{~S}_{1.4 \mathrm{GHz}} \gtrsim 260 \mu \mathrm{Jy}\right.$; Hodge et al. 2011), and COSMOS (2 $\mathrm{deg}^{2}$, $\mathrm{S}_{1.4 \mathrm{GHz}} \gtrsim 12 \mu \mathrm{Jy}$; Schinnerer et al. 2007, 2010; Lilly et al. 2007, 2009). The bottom panel shows the distribution of radio loudness, $R^{\prime}=0.4(i-t)$, for quasars in these three surveys.

\section{Radio-loud AGN: Relevance for feedback in massive galaxy formation}

By now, negative AGN feedback has become a standard ingredient in semi-analytic models and is required to reproduce the observed galaxy properties (e.g. Granato et al. 2004; Croton et al. 2006; Bower et al. 2006; Sijacki et al. 2007). In the models, this type of feedback, referred to as radio-mode feedback, is related to radio AGN outflows as the main source that heats the gas halo surrounding a massive galaxy. This heating thereby quenches the star formation and limits growth, thus avoiding the creation of overly high-mass galaxies. A detailed description of radio feedback is given in McAlpine et al. (2015).

The first observational support for AGN feedback was found using the combination of radio and X-ray data by McNamara et al. (2000), and Best et al. (2006) quantitatively showed that, in the local Universe, radio outflows may indeed balance the radiative cooling of the hot gas surrounding elliptical galaxies. Furthermore, it has been both theoretically postulated and observationally supported that this 'radio-mode' heating occurs during a quiescent phase of black-hole accretion 
(presumably via advection dominated accretion flows) and manifests as low-power radio AGN activity $\left(\mathrm{L}_{1.4 \mathrm{GHz}}<10^{25} \mathrm{~W} / \mathrm{Hz}\right.$; Evans et al. 2006; Hardcastle et al. 2006, 2007; Kauffmann et al. 2008; Smolčić et al. 2009a; Smolčić 2009; Smolčić \& Riechers 2011). Such low-power radio AGN, and the evolution of their comoving volume density (i.e. their radio luminosity function) through cosmic times, can be studied in detail only via simultaneously deep and large radio surveys with supplementary panchromatic data (Sadler et al. 2007; Donoso et al. 2009; Smolčić et al. 2009b; Simpson et al. 2012; McAlpine et al. 2013).

To date, the $20 \mathrm{~cm}$ radio luminosity function for low radio power $\mathrm{AGN}\left(\mathrm{L}_{1.4 \mathrm{GHz}} \lesssim 10^{25} \mathrm{~W} / \mathrm{Hz}\right)$ has been mostly derived out to $z=1.3$ (Sadler et al. 2007; Donoso et al. 2009; Smolčić et al. 2009b; Simpson et al. 2012; McAlpine et al. 2013). This also provided the first direct, radio-based, (albeit uncertain) observational support for radio-mode AGN feedback beyond the local universe (Smolčić et al. 2009b). This is illustrated in Figures 4 and 5. In Fig. 4 we show the $1.4 \mathrm{GHz}$ radio luminosity functions for red, quiescent galaxies drawn from the COSMOS two square degree survey (Scoville et al. 2007; Ilbert et al. 2010) out to $z=3$ (Smolčić et al., in prep). The luminosity functions were derived from the VLA-COSMOS 1.4 GHz Large Project (Schinnerer et al. 2007) reaching an rms of 10(15) $\mu \mathrm{Jy} / \mathrm{beam}$ over an area of 1(2) $\mathrm{deg}^{2}$. Stacking of the red, quiescent host galaxy population, selected following Ilbert et al. (2010), in the radio map constrained the luminosity function beyond $z=1.3$. The monochromatic luminosity was then converted to a kinetic power via scaling relations drawn from Bîrzan et al. (2008) and O'Sullivan et al. (2011). Integrating over the kinetic power averaged over comoving volume then yielded the heating rate exerted by radio luminous AGN onto their surroundings as a function of cosmic time out to $z=3$, as shown in Fig. 5 (see e.g. Smolčić et al. 2009b, for details). We stress that the result strongly depends on the i) knowledge of the low-luminosity end of the RL AGN luminosity function, and ii) conversion between monochromatic radio luminosity and kinetic power. As shown in the top panel of Fig. 5 when using the Bîrzan et al. scaling relation to convert between monochromatic radio luminosity and kinetic power the overall heating curve systematically rises depending on the low-luminosity boundary applied to the integral. This clearly illustrates the importance of both, i) constraining the conversion between monochromatic radio luminosity and kinetic power, and ii) constraining the luminosity functions of low-power radio AGN with high precision, especially at the low-luminosity end and out to the highest redshifts possible. The first is described in more detail in Kapińska et al. (2015), and the latter, a topic of this Chapter, will be possible to resolve with the SKA1 deep and wide continuum surveys, in conjunction with deep multi-wavelength data-sets. The assumed $5 \sigma$ limits of the wide and deep SKA1 surveys correspond to a $1.4 \mathrm{GHz}$ luminosity limit of $10^{23} \mathrm{~W} / \mathrm{Hz}$ (wide) and $2 \times 10^{22} \mathrm{~W} / \mathrm{Hz}$ (deep) at $z=2$, and $10^{24} \mathrm{~W} / \mathrm{Hz}$ (wide) and $2 \times 10^{23} \mathrm{~W} / \mathrm{Hz}$ (deep) at $z=6$. This will allow a direct examination of the faint end of the radio AGN luminosity function. Stacking in the radio maps will push this limit even further, while SKA2 will provide an order of magnitude push in sensitivity and determination of the faint end of the radio AGN luminosity function, as illustrated in Figs. 6 and 7.

\section{Studying AGN activity over cosmic time with the SKA}

SKA1 will provide a vital contribution to the science cases described above. Based on the SKADS (Wilman et al. 2008, 2010) simulation the SKA1 wide survey (assuming an rms of 


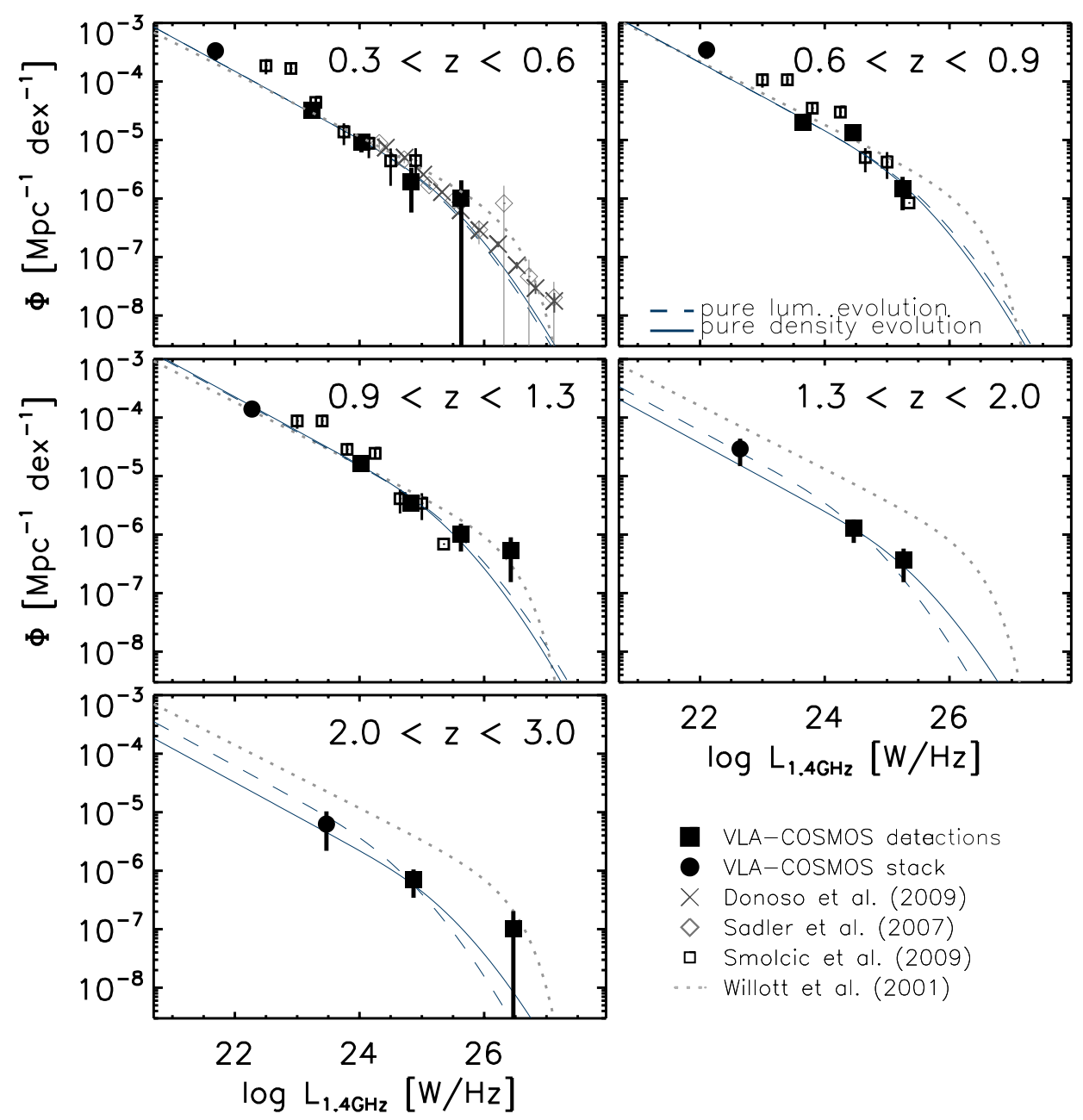

Figure 4: Radio luminosity functions (LFs) in five redshift bins out to $z=3$ for quiescent galaxies in the COSMOS field with stellar masses $\mathrm{M}_{*} \gtrsim 3 \times 10^{10} \mathrm{M}_{\odot} \mathrm{yr}^{-1}$ (Ilbert et al. 2010, filled circles and squares). The volume densities derived based on a volume limited radio detected sample are shown by the filled black squares, while those based on stacked data are shown by the filled black circles. The (full and dashed) lines show the best fit evolution to the COSMOS data in a given redshift range (blue curves) using the Sadler et al. (2002) local LF (dashed line: pure luminosity evolution; full line: pure density evolution). Various results from the literature, indicated in the legend, are also shown.

$1 \mu \mathrm{Jy} / \mathrm{beam}$ over $5000 \mathrm{deg}^{2}$ achieved in 1 year of observations) is expected to detect about $3 \times 10^{7}$, while the SKA1 deep survey (assuming an rms of $0.2 \mu \mathrm{Jy} / \mathrm{beam}$ over $30 \mathrm{deg}^{2}$ for 2000 hours of observations) is expected to detect $4 \times 10^{5} \mathrm{AGN}$ at $1 \mathrm{GHz}$. SKA2 will provide $10 \times$ higher sensitivities at this frequency. The results discussed above can serve as a path-finder for SKA survey planning, outlined as follows:

- At flux densities $\lesssim 100 \mu \mathrm{Jy}$, most sources will be SFGs and most AGN will be of the radioquiet type. Since the radio emission of these two classes may be powered by the same 


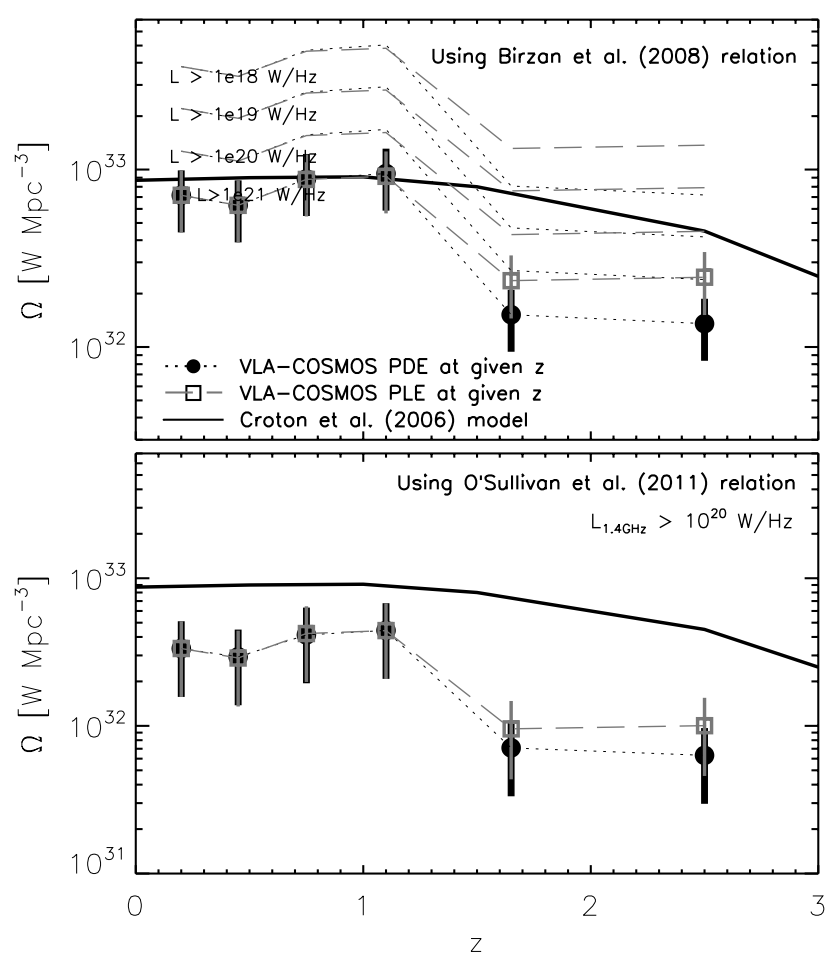

Figure 5: Comoving volume averaged heating rate $(\Omega)$ performed by quiescent massive galaxies in the COSMOS field as a function of redshift. $\Omega$ was derived by integrating the kinetic power per comoving volume over radio luminosity (see Smolčić et al. 2009b, for details). The COSMOS data points were derived by assuming pure density (filled circles), and pure luminosity (open squares) evolution best fit to the data in a given redshift bin. The upper and lower panels show $\Omega$ when using the relation between kinetic and 1.4 GHz radio luminosity from Bîrzan et al. (2008) and O'Sullivan et al. (2011), respectively. The (dotted and dashed) lines in the upper panel illustrate the discrepancy in $\Omega$ if various lower limit integral values are assumed (indicated in the panel). No such discrepancy is present when using the O'Sullivan et al. (2011) relation. The thick solid line in both panels shows the 'radio-mode feedback' heating rate drawn from the Croton et al. (2006) cosmological model, and required to reproduce observed galaxy properties.

mechanism pure continuum radio observations alone will not be able to distinguish between these populations;

- The classification of SKA radio sources will require ancilliary, multi-wavelength information. The location of the SKA surveys will therefore need to be planned carefully, in conjunction with existing and planned multi-wavelength surveys;

- The radio band, at sensitivities that will be provided by the SKA, carries the potential of becoming the optimal band to study the evolution of the most common types of AGN (the radio-quiet ones) given that radio emission is simultaneously insensitive to dust and provides high angular resolution, contrary to most other wavelength regimes;

- The SKA1 wide and deep surveys, in conjunction with multi-wavelength data will provide the basis to resolve the long-standing quasar radio-loudness dichotomy, unambiguously de- 

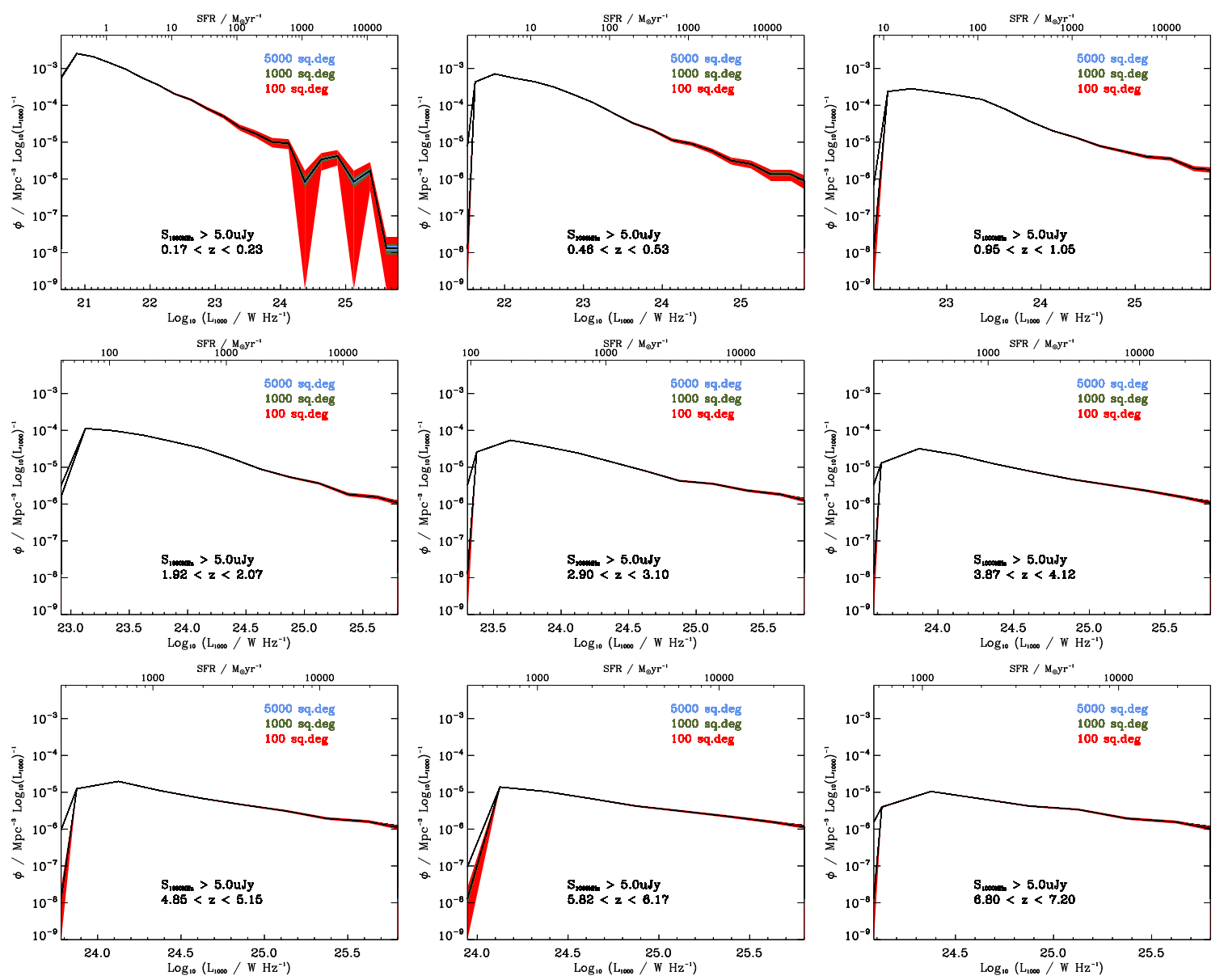

Figure 6: Radio AGN luminosity functions in various redshift bins (indicated in the panels) predicted for the wide SKA1 tier based on the SKADS simulations (Wilman et al. 2008, 2010).

termine the source of radio-emission in RQ AGN, and study the cosmic evolution of faint radio $\mathrm{AGN}$ out to the highest redshifts $(z \sim 6)$;

- Radio surveys have reached such depths that they are now dominated by the same galaxies detected by IR, optical and X-ray surveys. As a result, the SKA radio surveys will be an increasingly important component of multi-wavelength studies of galaxy formation and evolution and will therefore be useful to a very broad community.

\section{Acknowledgments}

VS acknowledges support from the European Union's Seventh Frame-work program under grant agreements 337595 and 333654. MJJ and MV acknowledge support by the South African Square Kilometre Array Project, the South African National Research Foundation. J.A. gratefully acknowledges support from the Science and Technology Foundation (FCT, Portugal) through the research grant PTDC/FIS-AST/2194/2012 and PEst-OE/FIS/UI2751/2014. 

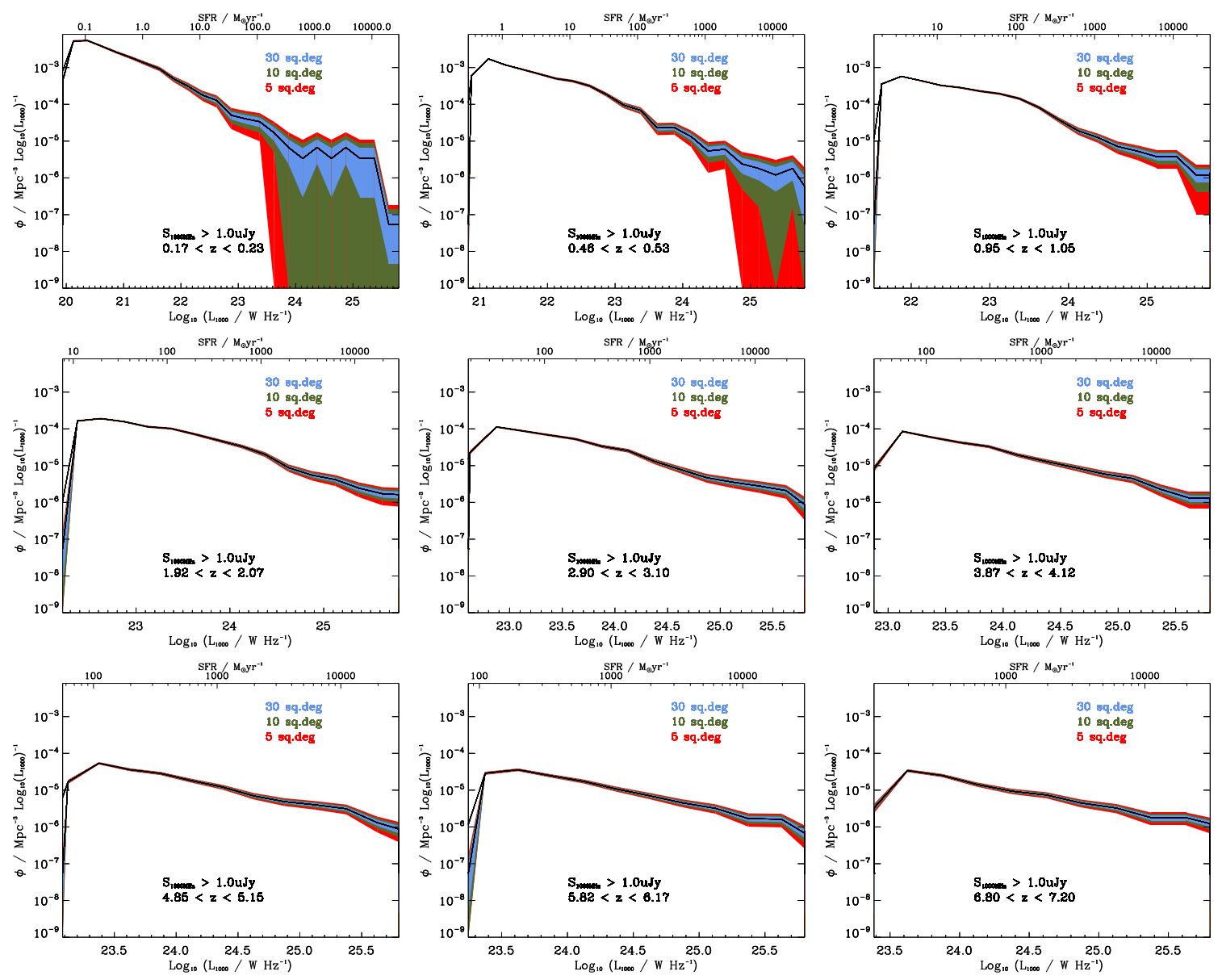

Figure 7: Radio AGN luminosity functions in various redshift bins (indicated in the panels) predicted for the deep SKA1 tier based on the SKADS simulations (Wilman et al. 2008, 2010).

\section{References}

Baloković, M., Smolčić, V., Ivezić, Ž., et al. 2012, ApJ, 759, 30

Best, P. N., \& Heckman, T. M. 2012, MNRAS, 421, 1569

Best, P. N., Kaiser, C. R., Heckman, T. M., \& Kauffmann, G. 2006, MNRAS, 368, L67

Biggs, A. D., \& Ivison, R. J. 2006, MNRAS, 371, 963

Bîrzan, L., McNamara, B. R., Nulsen, P. E. J., Carilli, C. L., \& Wise, M. W. 2008, ApJ, 686, 859

Bondi, M., Ciliegi, P., Schinnerer, E., et al. 2008, ApJ, 681, 1129

Bondi, M., Ciliegi, P., Zamorani, G., et al. 2003, A\&A, 403, 857

Bonzini, M., Padovani, P., Mainieri, V., et al. 2013, MNRAS, 436, 3759

Bonzini, M., Mainieri, V., Padovani, P., et al. 2012, ApJSS, 203, 15

—. submitted, MNRAS

Bower, R. G., Benson, A. J., Malbon, R., et al. 2006, MNRAS, 370, 645

Cirasuolo, M., Magliocchetti, M., Celotti, A., \& Danese, L. 2003, MNRAS, 341, 993

Condon, J. J., Kellermann, K. I., Kimball, A. E., Ivezić, Ž., \& Perley, R. A. 2013, ApJ, 768, 37

Condon, J. J., Cotton, W. D., Fomalont, E. B., et al. 2012, ApJ, 758, 23 
Croton, D. J., Springel, V., White, S. D. M., et al. 2006, MNRAS, 365, 11

Daddi, E., Alexander, D. M., Dickinson, M., et al. 2007, ApJ, 670, 173

de Ruiter, H. R., Zamorani, G., Parma, P., et al. 1997, A\&A, 319, 7

Del Moro, A., Alexander, D. M., Mullaney, J. R., et al. 2013, A\&A, 549, A59

Donoso, E., Best, P. N., \& Kauffmann, G. 2009, MNRAS, 392, 617

Dunlop, J. S., McLure, R. J., Kukula, M. J., et al. 2003, MNRAS, 340, 1095

Evans, D. A., Worrall, D. M., Hardcastle, M. J., Kraft, R. P., \& Birkinshaw, M. 2006, ApJ, 642, 96

Faber, S. M., Willmer, C. N. A., Wolf, C., et al. 2007, ApJ, 665, 265

Fabian, A. C. 2012, ARAA, 50, 455

Fanidakis, N., Baugh, C. M., Benson, A. J., et al. 2011, MNRAS, 410, 53

Fernandes, C. A. C., Jarvis, M. J., Rawlings, S., et al. 2011, MNRAS, 411, 1909

Fomalont, E. B., Kellermann, K. I., Cowie, L. L., et al. 2006, ApJSS, 167, 103

Granato, G. L., De Zotti, G., Silva, L., Bressan, A., \& Danese, L. 2004, ApJ, 600, 580

Gruppioni, C., Pozzi, F., Zamorani, G., et al. 2003, MNRAS, 341, L1

Gruppioni, C., Pozzi, F., Zamorani, G., \& Vignali, C. 2011, MNRAS, 416, 70

Hardcastle, M., Evans, D., \& Croston, J. 2007, MNRAS, 376, 1849

Hardcastle, M. J., Evans, D. A., \& Croston, J. H. 2006, MNRAS, 370, 1893

Hickox, R. C., Jones, C., Forman, W. R., et al. 2009, ApJ, 696, 891

Hodge, J. A., Becker, R. H., White, R. L., Richards, G. T., \& Zeimann, G. R. 2011, AJ, 142, 3

Hopkins, A., Georgakakis, A., Cram, L., Afonso, J., \& Mobasher, B. 2000, ApJSS, 128, 469

Hopkins, A. M., Afonso, J., Chan, B., et al. 2003, AJ, 125, 465

Hopkins, P. F., Somerville, R. S., Hernquist, L., et al. 2006, ApJ, 652, 864

Huynh, M. T., Jackson, C. A., Norris, R. P., \& Prandoni, I. 2005, AJ, 130, 1373

Ibar, E., Ivison, R. J., Biggs, A. D., et al. 2009, MNRAS, 397, 281

Ilbert, O., Salvato, M., Le Floc'h, E., et al. 2010, ApJ, 709, 644

Ivezić, Ž., Menou, K., Knapp, G. R., et al. 2002, AJ, 124, 2364

Jarvis, M. J., \& Rawlings, S. 2004, New Astronomy Reviews, 48, 1173

Kapińska, A. D., Hardcastle, M. J., Jackson, C. A., et al. 2015, "Unravelling lifecycles and physics of radio-loud AGN in the SKA Era", in "Advancing Astrophysics with the Square Kilometre Array", POS (AASKA14) 173

Karim, A., Schinnerer, E., Martínez-Sansigre, A., et al. 2011, ApJ, 730, 61

Kauffmann, G., Heckman, T. M., \& Best, P. N. 2008, MNRAS, 384, 953

Kimball, A. E., Kellermann, K. I., Condon, J. J., Ivezić, Ž., \& Perley, R. A. 2011, ApJL, 739, L29

Lilly, S. J., Le Fèvre, O., Renzini, A., et al. 2007, ApJSS, 172, 70

Lilly, S. J., Le Brun, V., Maier, C., et al. 2009, ApJSS, 184, 218

McAlpine, K., Jarvis, M. J., \& Bonfield, D. G. 2013, MNRAS, 436, 1084

McAlpine, K., Prandoni, I., Jarvis, M., et al. 2015, "The SKA view of the Interplay between SF and AGN Activity and its role in Galaxy Evolution", in "Advancing Astrophysics with the Square Kilometre Array", PoS (AASKA14) 083

McNamara, B. R., Wise, M., Nulsen, P. E. J., et al. 2000, ApJL, 534, L135

Mignano, A., Prandoni, I., Gregorini, L., et al. 2008, A\&A, 477, 459

Miller, N. A., Fomalont, E. B., Kellermann, K. I., et al. 2008, ApJSS, 179, 114

Miller, N. A., Bonzini, M., Fomalont, E. B., et al. 2013, ApJSS, 205, 13 
Miller, P., Rawlings, S., \& Saunders, R. 1993, MNRAS, 263, 425

Morić, I., Smolčić, V., Kimball, A., et al. 2010, ApJ, 724, 779

O'Sullivan, E., Giacintucci, S., David, L. P., et al. 2011, ApJ, 735, 11

Owen, F. N., \& Morrison, G. E. 2008, AJ, 136, 1889

Padovani, P., Mainieri, V., Tozzi, P., et al. 2009, ApJ, 694, 235

Padovani, P., Miller, N., Kellermann, K. I., et al. 2011, ApJ, 740, 20

Prandoni, I., Gregorini, L., Parma, P., et al. 2001, A\&A, 365, 392

Prandoni, I., \& Seymour, N. 2015, "Revealing the Physics and Evolution of Galaxies and Galaxy Clusters with SKA Continuum Surveys", in "Advancing Astrophysics with the Square Kilometre Array", POS (AASKA14) 067

Roy, A. L., Norris, R. P., Kesteven, M. J., Troup, E. R., \& Reynolds, J. E. 1998, MNRAS, 301, 1019

Sadler, E. M., Jackson, C. A., Cannon, R. D., et al. 2002, MNRAS, 329, 227

Sadler, E. M., Cannon, R. D., Mauch, T., et al. 2007, MNRAS, 381, 211

Sanders, D. B., \& Mirabel, I. F. 1996, ARAA, 34, 749

Schinnerer, E., Smolčić, V., Carilli, C. L., et al. 2007, ApJSS, 172, 46

Schinnerer, E., Sargent, M. T., Bondi, M., et al. 2010, ApJSS, 188, 384

Schneider, D. P., Richards, G. T., Hall, P. B., et al. 2010, AJ, 139, 2360

Scoville, N., Aussel, H., Brusa, M., et al. 2007, ApJSS, 172, 1

Seymour, N., McHardy, I. M., \& Gunn, K. F. 2004, MNRAS, 352, 131

Seymour, N., Dwelly, T., Moss, D., et al. 2008, MNRAS, 386, 1695

Shabala, S. S., Kaviraj, S., \& Silk, J. 2011, MNRAS, 413, 2815

Sijacki, D., Springel, V., Di Matteo, T., \& Hernquist, L. 2007, MNRAS, 380, 877

Simpson, C., Martínez-Sansigre, A., Rawlings, S., et al. 2006a, MNRAS, 372, 741

-. 2006b, MNRAS, 372, 741

Simpson, C., Rawlings, S., Ivison, R., et al. 2012, MNRAS, 421, 3060

Smolčić, V. 2009, ApJL, 699, L43

Smolčić, V., \& Riechers, D. A. 2011, ApJ, 730, 64

Smolčić, V., Schinnerer, E., Scodeggio, M., et al. 2008, ApJSS, 177, 14

Smolčić, V., Zamorani, G., Schinnerer, E., et al. 2009a, ApJ, 696, 24

Smolčić, V., Schinnerer, E., Zamorani, G., et al. 2009b, ApJ, 690, 610

Sopp, H. M., \& Alexander, P. 1991, MNRAS, 251, 14P

Strittmatter, P. A., Hill, P., Pauliny-Toth, I. I. K., Steppe, H., \& Witzel, A. 1980, A\&A, 88, L12

Ulvestad, J. S., Antonucci, R. R. J., \& Barvainis, R. 2005, ApJ, 621, 123

White, R. L., Becker, R. H., Helfand, D. J., \& Gregg, M. D. 1997, ApJ, 475, 479

White, R. L., Helfand, D. J., Becker, R. H., Glikman, E., \& de Vries, W. 2007, ApJ, 654, 99

White, R. L., Becker, R. H., Gregg, M. D., et al. 2000, ApJSS, 126, 133

White, S. V., Jarvis, M. J., Häußler, B., \& Maddox, N. 2014, ArXiv e-prints, arXiv:1410.3892

Wilman, R. J., Jarvis, M. J., Mauch, T., Rawlings, S., \& Hickey, S. 2010, MNRAS, 405, 447

Wilman, R. J., Miller, L., Jarvis, M. J., et al. 2008, MNRAS, 388, 1335

Zakamska, N. L., \& Greene, J. E. 2014, MNRAS, 442, 784

Zwart, J. T. L., Jarvis, M. J., Deane, R. P., et al. 2014, MNRAS, 439, 1459 